\section{Educación geográfica y ambiental: Percepción de riesgo por peligros hidrometeorológicos en la comunidad.}

Geographic and environmental education: Perception of risk for hydrometeorological hazards in the community.

Educação geográfica e ambiental: percepção de risco de riscos hidrometeorológicos na comunidade.

Recibido: 06/01/2020

Aceptado: $18 / 05 / 2020$

Publicado: 30/06/2020

\title{
RESUMEN
}

La Geografía y el estudio del subjetivismo han revelado que el imaginario territorial tiene gran importancia en el comportamiento espacial del ser humano, en la territorialidad donde existe cada individuo, reguladora de sus prácticas sociales. El presente trabajo está dirigido a revelar la contribución del conocimiento geográfico local a la percepción de riesgo por peligros hidrometeorológicos extremos, en el entorno diario de las personas. Se concibe, diseña y valida un proyecto de intervención educativa con un grupo de estudio, en torno a su comunidad, aplicándose el método sicométrico como aproximación metodológica, para entender algunas de las actuaciones del sujeto como fundamento de la vulnerabilidad ante el peligro concreto que se analiza. Se identifican las pautas cognoscitivas generales para la concepción, el diseño y la implementación de una estrategia educativa geográfica, por demás ambiental, susceptible a su adaptación según los contextos, la cual está dirigida a la formación cultural ambiental ciudadana, capaz de orientar los modos de actuación cotidianos hacia los caminos de la sostenibilidad.

PALABRAS CLAVE: educación geográfica, educación ambiental, percepción, riesgo de peligros naturales

\section{ABSTRACT}

Geography and the study of subjectivism have revealed that the territorial imaginary has great importance in the spatial behavior of the human being, in the territoriality where each individual exists, regulator of their social practices. The present work is aimed at revealing the contribution of local geographic knowledge to the perception of risk from extreme hydro meteorological hazards, in the daily environment of people. An educational intervention project is conceived, designed and validated with a study group, around their community, applying the psychometric method as a methodological approach, to understand some of the subject's actions as the basis of vulnerability to the specific danger that is analyze. The general cognitive guidelines are identified for the conception, design and implementation of a geographic educational strategy, which is also environmental, susceptible to its adaptation according to the contexts, which is aimed at the environmental citizen cultural training, capable of guiding the ways of daily actions towards the paths of sustainability.

KEYWORDS: geographic education, environmental education, perception, risk of natural hazards 


\section{RESUMO}

A geografia e o estudo do subjetivismo revelaram que o imaginário territorial tem grande importância no comportamento espacial do ser humano, na territorialidade em que cada indivíduo existe, regulador de suas práticas sociais. $O$ presente trabalho tem como objetivo revelar a contribuição do conhecimento geográfico local para a percepção de risco de perigos hidrometeorológicos extremos, no ambiente cotidiano das pessoas. Um projeto de intervenção educacional é concebido, projetado e validado com um grupo de estudo, em torno de sua comunidade, aplicando o método psicométrico como uma abordagem metodológica, para entender algumas das ações do sujeito como a base da vulnerabilidade ao perigo específico. analisar. São identificadas as diretrizes cognitivas gerais para a concepção, desenho e implementação de uma estratégia educacional geográfica, também ambiental, suscetível de adaptação de acordo com os contextos, voltada ao treinamento cultural ambiental do cidadão, capaz de orientar os modos de ações diárias em direção aos caminhos da sustentabilidade.

PALAVRAS CHAVE: educação geográfica, educação ambiental, percepção, risco de riscos naturais

\section{INTRODUCCIÓN}

El conocimiento geográfico como reflejo subjetivo del mundo objetivo, del nexo de interpretación de los objetos, fenómenos y procesos geográficos concurrentes en la superficie terrestre, en el contexto de la relación Naturaleza-Sociedad; se configura como fundamento para la asimilación territorial y para la gestión ambiental sostenible de los subsistemas natura-sociales a escala regional y local, propiciando la satisfacción de sus funciones informativa, instrumental y transformadora de la realidad social (Mateo, 2001). Esta reflexión reafirma la trascendencia del conocimiento geográfico para comprender "el carácter de los lugares (...) en el contexto de un lugar específico» (Unión Geográfica Internacional/Comisión de Educación Geográfica, 1992, s/p), en relación al espacio-geográfico de producción/reproducción de la vida social donde concurren los riesgos por peligros hidrometeorológicos extremos, objeto de estudio en la presente investigación.

La ocupación, apropiación y transformación del espacio geográfico es un proceso cultural, porque se crean bienes materiales en torno a las relaciones sociales, valores, modos de hacer, de pensar y de percibir el mundo, todo lo cual constituye el patrimonio cultural construido por la humanidad a lo largo de la historia.

La Geografía' siempre ha considerado la complejidad de su "objeto» al tratar nada menos que toda una dimensión de la realidad, independientemente de las reducciones del campo de estudio del cual se trate; por ello ha desarrollado una amplia diversidad de líneas de pensamiento, que han respondido a visiones ideológico-filosóficas particulares, tales como la mecanicista, la racionalista, la sistémica y la holista. Cada una de ellas ha visualizado lo ambiental desde una perspectiva particular, priorizando determinadas tradiciones geográficas y elaborando las categorías de análisis particulares a través de múltiples corrientes: determinismo, corológico-regional, posibilismo, teórico-cuantitativa, humanística, crítica, y ambiental (Lobato, 1995).

La conexión entre educación ambiental y gestión de riesgos han de armonizar en la promoción de un conocimiento adecuado del entorno en el cual se vive, en la valoración real de este y en el reconocimiento de las especificidades que contiene, pues se trata fundamentalmente de prevenir, de lograr una buena y armónica relación con el espacio circundante.

\footnotetext{
${ }^{1}$ La historia, concepción teórica, filosófica y científica de la Geografía ha configurado extensos legajos impresos y de oratoria, a lo largo de la historia humana. Una síntesis de La Geografía como ciencias se describe en el tabloide del Curso Universidad para Todos de Geografía.
} 
La presente investigación-dentro de la vulnerabilidad social-aporta al análisis de la percepción del riesgo por peligros hidrometeorológicos extremos (fuertes vientos, inundaciones por intentas lluvias o penetraciones del mar) en Cuba, considerando los resultados del estudio de percepción realizado por el Grupo de Evaluación de Riesgos de la Agencia de Medio Ambiente, al cual se le incorporó la dimensión geográfica (relieve y paisajes) en la interpretación de los resultados de la entrevista aplicada a 16626 individuos del país, y el proceso de gestión-capacitación educativa en temas relacionados con las ciencias geográficas (por demás ambiental), a los actores-gestores locales del territorio correspondiente al estudio de caso.

La situación problémica gira en torno a la siguiente pregunta: ¿Cómo el conocimiento geográfico contribuye a elevar la percepción de riesgo por peligros hidrometeorológicos extremos del individuo, en los espacios de producción-reproducción social de vida cotidiana?

Desde esta perspectiva se expone y valida la propuesta de concepción propositiva de la educación geográfica en el entorno de vida cotidiana del sujeto, dirigida a revelar la contribución de la misma en la formación cultural ambiental para la percepción de riesgos hidrometeorológicos extremos.

La metodología utilizada combina la intervinculación metodológica y teórica de las perspectivas de análisis provenientes de la geografía, la filosofía de la ciencia, los estudios en ciencia, tecnología y sociedad, así como los estudios de percepción y comunicación del riesgo en los marcos del desarrollo local sostenible; en particular, incluye los estudios de percepción de peligro (fuertes vientos, inundaciones por intensas lluvias o penetraciones del mar) del Grupo de Evaluación de Riesgos de la Agencia de Medio Ambiente (2009-2011), a partir de una entrevista estructurada a 16626 individuos $^{2}$ de todo el país.

Son analizados los datos de percepción obtenidos en el estudio de referencia, relacionándolos con las particularidades geográficas generales de los municipios según el tipo predominante de relieve (llanura-altura-montaña), de paisaje geográfico y el grado de inundación costera, por unidad territorial: país, región ${ }^{3}$, provincia y municipio. Estos resultados se correlacionan con los datos referentes al grado de conocimiento del sujeto y sus vulnerabilidades, y en la adopción de las mejores opciones de comportamiento antes, durante y posterior a la ocurrencia del/los fenómenos(s) meteorológico(s) de estudio, que ofrezcan criterios para el diseño e implementación de acciones educativas para su aprehensión social.

Se procedió al cruzamiento de la información mediante tablas de contingencia y de frecuencia por unidad territorial descrita, con variables sociodemográficas tales como sexo, edad, nivel de instrucción, ocupación, entre otras; y por rasgos geográficos del municipio de residencia de los encuestados. Los datos fueron procesados a través del programa estadístico computarizado IBM SPSS v.22 (Statistic Package Social Sciences). Se elaboraron perfiles de riesgo siguiendo la norma del paradigma sicométrico.

Los niveles empíricos de análisis y síntesis de la dimensión geográfica de la base de datos nacional (Agencia de Medio Ambiente [AMA], 2013), de acuerdo con el esquema de análisis teóricometodológico concebido, se objetivaron a partir de la interpretación de las variables: a) conocimiento del peligro que más afecta al sujeto; b) reconocimiento de la causa principal que este le atribuye a las afectaciones; c) actuación del sujeto ante el peligro -¿qué hizo para protegerse?-; d) nivel de evaluación,

\footnotetext{
${ }^{2}$ La encuesta se aplicó en todas las provincias del país, exceptuando la provincia La Habana, con una muestra representativa para las provincias, los municipios y los consejos populares.

${ }^{3}$ Se asume el criterio que establece el Instituto de Meteorología de Cuba: Región Occidental (Pinar del Río, Artemisa, La Habana, Mayabeque, Matanzas e Isla de la Juventud; Región Central: Villa Clara, Cienfuegos, Sancti Spíritus y Ciego de Ávila; Región Oriental: Camagüey, Las Tunas, Holguín, Granma, Santiago de Cuba y Guantánamo.
} 
por el sujeto, de las afectaciones relacionadas con el peligro de estudio, principalmente de su ámbito de vida cotidiana; e) grado de conocimiento para minimizar afectaciones y los modos de actuación ante la inminencia del/los peligro/s hidrometeorológico/s extremo/s; y f) nivel de actuación proactiva o no del sujeto, actitud para la prevención del peligro; de los cuales se infieren la comprensión de la subjetividad del indagado y su correlación empírica con relación al grado de conocimiento del peligro, de los modos de actuación ante los mismos u otros aspectos, base para la distinción de la percepción de riesgo por peligros hidrometeorológicos extremos.

\section{DESARROLLO}

El estudio social del riesgo y los desastres asociados a las actividades humanas o a amenazas naturales, ha sido de gran importancia para los científicos sociales, no solo para aquellos que se encargan del estudio del comportamiento humano, como los sicólogos o sociólogos, sino también para los de otras disciplinas como los geógrafos, antropólogos, historiadores, etc.

Mencionan Conesa y Calvo (2003) que el riesgo tiene una dimensión espacial porque se presenta en un territorio determinado, y es justo el análisis espacial la clave del trabajo del geógrafo. La vocación de cartografiar los espacios y el tipo de riesgo que amenaza al lugar, desde la visión sistémica de los condicionantes naturales y sociales contextualmente, permite elaborar políticas de gestión basadas sobre todo en el concepto de riesgo aceptable.

El diseño de una estrategia de educación geográfica local que promueva procesos de enseñanzaaprendizaje acerca de las particularidades territoriales y la dinámica de los sistemas naturales, en este caso, el paisaje geográfico donde transcurre la vida cotidiana del sujeto, dirigido a elevar la percepción de riesgos por peligros hidrometeorológicos extremos; constituye uno de los objetivos de la investigación en su diseño teórico. No obstante, su alcance es más abarcador en el ámbito de la educación ambiental para el desarrollo sostenible local y la reducción de riesgos en el amplio sentido del término, como contribución al fomento de una cultura de prevención apropiada al contexto de vida social (Almaguer, 2008; Castañeda, 2014).

En la Estrategia Ambiental Nacional (2010-2015), se plantea a la educación ambiental como uno de los instrumentos de la política y la gestión ambiental. Este presupuesto promueve el desenvolvimiento de procesos de intervención educativa y de comunicación ambiental al concebir la incorporación de los resultados de los estudios de percepción de vulnerabilidad y riesgos, para el desarrollo de capacidades de gestión educativa en todos los actores-gestores de la sociedad, aunque no explicita la necesaria sensibilización y percepción de estos riesgos y peligros en torno al sujeto social territorialmente, como se aborda en la presente investigación.

Para valorar el conocimiento geográfico en la percepción de riesgo por peligros de fuertes vientos, inundaciones por intensas lluvias y penetraciones del mar, en los espacios de asimilación de producción/reproducción de vida cotidiana del individuo, se requiere concebir el conocimiento geográfico local-como ciencia y como enseñanza- contextualizado al territorio y a la multiplicidad de actores y sectores sociales que lo configuran, teniendo en cuenta la Teoría de los Paisajes como fundamento para el estudio de la localidad, asumiéndose el paisaje como objeto sintetizador de la geografía local.

En este sentido, la estrategia de educación geográfica se concibe como una propuesta de concepción teórico-metodológica y educativo general para proceso de enseñanza-aprendizaje de la geografía local de vida cotidiana del individuo, lo que ofrece pautas generales para su posible aplicación según el territorio y los actores involucrados contextualmente. Consecuentemente, ello se articula con los resultados de la entrevista en profundidad realizada a informantes clave del territorio: representantes del gobierno, del centro de gestión de riesgos, de la universidad, de instituciones sociales, etc.; acerca 
de la pertinencia de educar a la población en el conocimiento de su entorno de vida y la percepción de los riesgos de estudio. Lo esencial es mostrar las relaciones del ser humano con su ambiente inmediato, potenciando la formación de valores y modos de actuación adaptados a las condiciones ambientales a las cuales se puede enfrentar, en particular, la percepción de riesgos por peligros hidrometeorológicos extremos u otros similares.

El eje que concierne a la Geografía local, unidad V «Nuestro mundo de vida social local» constituye el fundamento de la propuesta de la presente investigación, la cual se concibe y constata empíricamente en un grupo de estudio del municipio Mariel a través de un curso de posgrado sobre los conocimientos elementales a tener en cuenta para el tratamiento de estudios ambientales locales, coordinado por el Departamento de Extensión Universitaria de la Cujae, y en otras acciones educativas desarrolladas con la Universidad de Artemisa.

Como parte del proceso de capacitación, se concibió una encuesta aplicada a los participantes del curso, como técnica del paradigma cuantitativo y con la metódica de la indagación cualitativa, dado el carácter de la percepción de riesgos por peligros hidrometeorológicos extremos de los individuos, que permite su aplicación en el estudio de un mismo fenómeno como parte de la triangulación metodológica.

Estos atributos-a criterio del autor-constituyen referentes generales que modelan la concepción de acciones educativas, de formación cultural general de capacidades y habilidades cognitivas, que redunden en la percepción de los peligros y pauten los modos de actuación individual y colectiva del sujeto en su accionar cotidiano (Bayón, 2014). Se aplica y enriquece contextualmente las metodologías de estudios aplicados por Puy (1994), que retoma la propuesta del Grupo de Oregón (Fischoff et al., 1978 y Slovic et al., 1980, 1985; como se citó en Puy, 1994); que incluye nueve atributos de valoración de las percepciones de riesgos, los cuales retoma Almaguer (2008) en localidades del oriente cubano.

En el trabajo que se presenta, se incluyen doce atributos que contemplan, además, los factores de aceptabilidad de riesgo, descritas por Velk y Stallen (1980), que se citó en Espluga, 2002 y se puede observar en la tabla 1.

Tabla 1. Escalas de atributos cualitativos de percepción de peligros hidrometeorológicos, para valorar la actitud del sujeto según escala de tipo Likert.

\begin{tabular}{|c|c|c|c|c|c|c|c|c|}
\hline \multirow{3}{*}{ Involuntario } & \multicolumn{7}{|c|}{ Voluntariedad del riesgo ante el peligro } & \multirow{3}{*}{ Voluntario } \\
\hline & \multicolumn{7}{|c|}{ II-1¿Se enfrenta usted a este peligro de forma voluntaria o involuntaria? } & \\
\hline & Riesgo Involuntario & 1 & 2 & 3 & 4 & 5 & $\begin{array}{c}\text { Riesgo } \\
\text { Voluntario }\end{array}$ & \\
\hline \multirow{3}{*}{ Inmediato } & \multicolumn{7}{|c|}{ Inmediatez del efecto } & \multirow{3}{*}{ Retardado } \\
\hline & \multicolumn{7}{|c|}{$\begin{array}{l}\text { II-2 En caso de ocurrir ¿cuándo se experimentarían los efectos más nocivos de } \\
\text { este peligro? }\end{array}$} & \\
\hline & Efecto Inmediato & 1 & 2 & 3 & 4 & 5 & $\begin{array}{c}\text { Efecto } \\
\text { Retardado }\end{array}$ & \\
\hline \multirow{3}{*}{$\begin{array}{l}\text { Desconocido } \\
\text { (cognitivo/ } \\
\text { vulnerable) }\end{array}$} & \multicolumn{7}{|c|}{ Conocimiento del riesgo ante el peligro } & \multirow{3}{*}{$\begin{array}{l}\text { Conocido } \\
\text { (cognitivo/ } \\
\text { no } \\
\text { vulnerable) }\end{array}$} \\
\hline & \multicolumn{7}{|c|}{$\begin{array}{l}\text { II-3 ¿En qué medida usted conoce el riesgo asociado a este peligro? (daños } \\
\text { que puede causarle, posibilidades que tiene de experimentar estos daños, } \\
\text { etc.) }\end{array}$} & \\
\hline & Nivel de riesgo Desconocido & 1 & 2 & 3 & 4 & 5 & $\begin{array}{l}\text { Nivel de riesgo } \\
\text { Conocido con } \\
\text { precisión }\end{array}$ & \\
\hline
\end{tabular}




\begin{tabular}{|c|c|c|c|c|c|c|c|c|}
\hline \multirow{3}{*}{$\begin{array}{l}\text { Desconocido } \\
\quad \text { (líderes- } \\
\text { comunidad/ } \\
\text { responsables) }\end{array}$} & \multirow{2}{*}{\multicolumn{7}{|c|}{$\begin{array}{l}\text { Conocimiento del riesgo (ciencia; instituciones; gestores comunitarios) } \\
\text { II-4 ¿En qué medida conoce la ciencia y los responsables de la comunidad } \\
\text { a este peligro? }\end{array}$}} & \multirow{3}{*}{$\begin{array}{l}\text { Conocido } \\
\text { (líderes- } \\
\text { comunidad/ } \\
\text { responsable) }\end{array}$} \\
\hline & & & & & & & & \\
\hline & Nivel de riesgo Desconocido & 1 & 2 & 3 & 4 & 5 & $\begin{array}{l}\text { Nivel de riesgo } \\
\text { Conocido con } \\
\text { precisión }\end{array}$ & \\
\hline \multirow{3}{*}{$\begin{array}{l}\text { No controlable } \\
\text { (percepción/ } \\
\text { vulnerable) }\end{array}$} & \multicolumn{7}{|c|}{ Control (personal) sobre el riesgo con relación al peligro } & \multirow{3}{*}{$\begin{array}{c}\text { Controlable } \\
\text { (percepción/ } \\
\text { no } \\
\text { vulnerable) }\end{array}$} \\
\hline & \multicolumn{7}{|c|}{$\begin{array}{l}\text { II-5 ¿Hasta qué punto usted puede, por habilidad personal o diligencia, } \\
\text { intervenir para controlar el daño que puede causarle este peligro? }\end{array}$} & \\
\hline & $\begin{array}{l}\text { El riesgo personal no se puede } \\
\text { controlar }\end{array}$ & 1 & 2 & 3 & 4 & 5 & $\begin{array}{l}\text { El riesgo } \\
\text { personal se } \\
\text { puede controlar }\end{array}$ & \\
\hline \multirow{3}{*}{ Nuevo } & \multicolumn{7}{|c|}{ Novedad del riesgo relacionado con el peligro } & \multirow{3}{*}{ Antiguo } \\
\hline & \multicolumn{7}{|c|}{$\begin{array}{l}\text { II-6 En términos de nuevo-novedoso o antiguo-familiar, este peligro es } \\
\text { para usted o su comunidad. }\end{array}$} & \\
\hline & Nuevo & 1 & 2 & 3 & 4 & 5 & Antiguo & \\
\hline \multirow{3}{*}{ No catastrófico } & \multicolumn{7}{|c|}{ Constante/individual-Catastrófico el riesgo que provoca el peligro } & \multirow{3}{*}{ Catastrófico } \\
\hline & \multicolumn{7}{|c|}{$\begin{array}{l}\text { II-7 ¿En qué grado este peligro que puede dañar a un gran número de } \\
\text { personas de una sola vez? }\end{array}$} & \\
\hline & Constante/individual & 1 & 2 & 3 & 4 & 5 & Catastrófico & \\
\hline \multirow{3}{*}{ No temido } & \multicolumn{7}{|c|}{ No temido-temido } & \multirow{3}{*}{ Temido } \\
\hline & \multicolumn{7}{|c|}{$\begin{array}{l}\text { II-8 ¿En qué grado usted ha aprendido a convivir con dicho peligro y no } \\
\text { teme, o es uno al que le tiene gran temor? }\end{array}$} & \\
\hline & No temido & 1 & 2 & 3 & 4 & 5 & Temido & \\
\hline \multirow{3}{*}{$\begin{array}{l}\text { Desconocido } \\
\text { (Entorno) }\end{array}$} & \multicolumn{7}{|c|}{$\begin{array}{c}\text { Conocimiento de vulnerabilidad del entorno de vida-individual y } \\
\text { colectiva- ante el riesgo del peligro }\end{array}$} & \multirow{3}{*}{$\begin{array}{l}\text { Conocido } \\
\text { (entorno) }\end{array}$} \\
\hline & \multicolumn{7}{|c|}{$\begin{array}{l}\text { II-9 ¿En qué medida usted conoce la exposición al riesgo asociado al } \\
\text { peligro en cuestión, según las características del entorno de vida } \\
\text { cotidiana? }\end{array}$} & \\
\hline & $\begin{array}{l}\text { Nivel de vulnerabilidad } \\
\text { Desconocido }\end{array}$ & 1 & 2 & 3 & 4 & 5 & $\begin{array}{l}\text { Nivel de } \\
\text { vulnerabilidad } \\
\text { Conocido }\end{array}$ & \\
\hline \multirow{3}{*}{$\begin{array}{l}\text { Desconocido } \\
\text { (vulnerabilidad } \\
\text { /sujeto) }\end{array}$} & \multicolumn{7}{|c|}{$\begin{array}{c}\text { Conocimiento de vulnerabilidad de sufrir daño personal de producirse el } \\
\text { evento peligroso }\end{array}$} & \\
\hline & \multicolumn{7}{|c|}{$\begin{array}{l}\text { II-10. La posibilidad de que Ud. experimente un daño como consecuencia } \\
\text { de este peligro es: }\end{array}$} & $\begin{array}{l}\text { Conocido } \\
\text { (vulnerabilida }\end{array}$ \\
\hline & $\begin{array}{l}\text { Nivel de vulnerabilidad } \\
\text { Desconocido }\end{array}$ & 1 & 2 & 3 & 4 & 5 & $\begin{array}{c}\text { Nivel de } \\
\text { vulnerabilidad } \\
\text { Conocido } \\
\end{array}$ & d/sujeto) \\
\hline & Gravedad d & As C & nise & cuer & cias & & & \\
\hline Poco grave & $\begin{array}{r}\text { II-11 En caso de producirse, la g } \\
\text { este }\end{array}$ & & & $\overline{e l d}$ & $\overline{\tilde{n} o c}$ & ue & puede causar & Muy grave \\
\hline & Seguro que no va a ser Mortal & 1 & 2 & 3 & 4 & 5 & $\begin{array}{l}\text { Seguro que va a } \\
\text { ser Mortal }\end{array}$ & \\
\hline & Autogestión & ries & 50 & el p & ligr & & & \\
\hline No controlable & $\begin{array}{r}\text { II-12. ¿En qué grado usted puede } \\
\text { situación de consecuencias d } \\
\text { recursc }\end{array}$ & & & & & & $\begin{array}{l}\text { esencadene una } \\
\text { ara su vida y }\end{array}$ & \\
\hline $\begin{array}{l}\text { (baja- } \\
\text { percepción) }\end{array}$ & $\begin{array}{l}\text { Nivel de vulnerabilidad } \\
\text { Desconocido }\end{array}$ & 1 & 2 & 3 & 4 & 5 & $\begin{array}{c}\text { Nivel de } \\
\text { vulnerabilidad } \\
\text { Conocido }\end{array}$ & Controlable \\
\hline
\end{tabular}

Fuente: Elaborada por el autor sobre la propuesta de Slovic et al. (1995), citado por Puy (1994). 
Se incluyeron las características socio-demográficas generales de los individuos entrevistados (edad, sexo, nivel de escolaridad y ocupación), pues en varios estudios se ha comprobado que existe cierta relación entre estas y las percepciones de peligros y riesgos, según la literatura consultada, que matizan la actitud o disposición sicológica de las personas a través de la propia experiencia (Slovic y Weber, 2002; Puy, 1994; Puy y Aragonés, 2003; Castañeda, 2014). Con la finalidad de medir las diferentes variables o atributos del riesgo en los sujetos indagados (cursistas que constituyen habitantes expuestos), se empleó el enfoque sicométrico antes mencionado, empleando la combinación de Escala del tipo Likert de 5 puntos, con un diferencial semántico, otorgándose 1 punto como puntuación mínima al ítem y 5 puntos a una respuesta que otorga el valor máximo al ítem propuesto. Esta medición indirecta (tabla 1), en las que partiendo de una serie de afirmaciones, proposiciones o juicios sobre los que los individuos manifiestan su opinión, se deducen las actitudes, inferidas de las expresiones verbales o de la conducta observada en el sujeto.

El análisis de los datos permite la creación del perfil característico de la percepción para cada tipo de peligro. La construcción gráfica de los diferentes perfiles ofrece una panorámica descriptiva de las valoraciones realizadas por los asistentes al curso, la cual puede ser contrastada con los resultados finales del mismo, como experiencia formativa (figura 1).

Figura 1. Perfiles de riesgo.

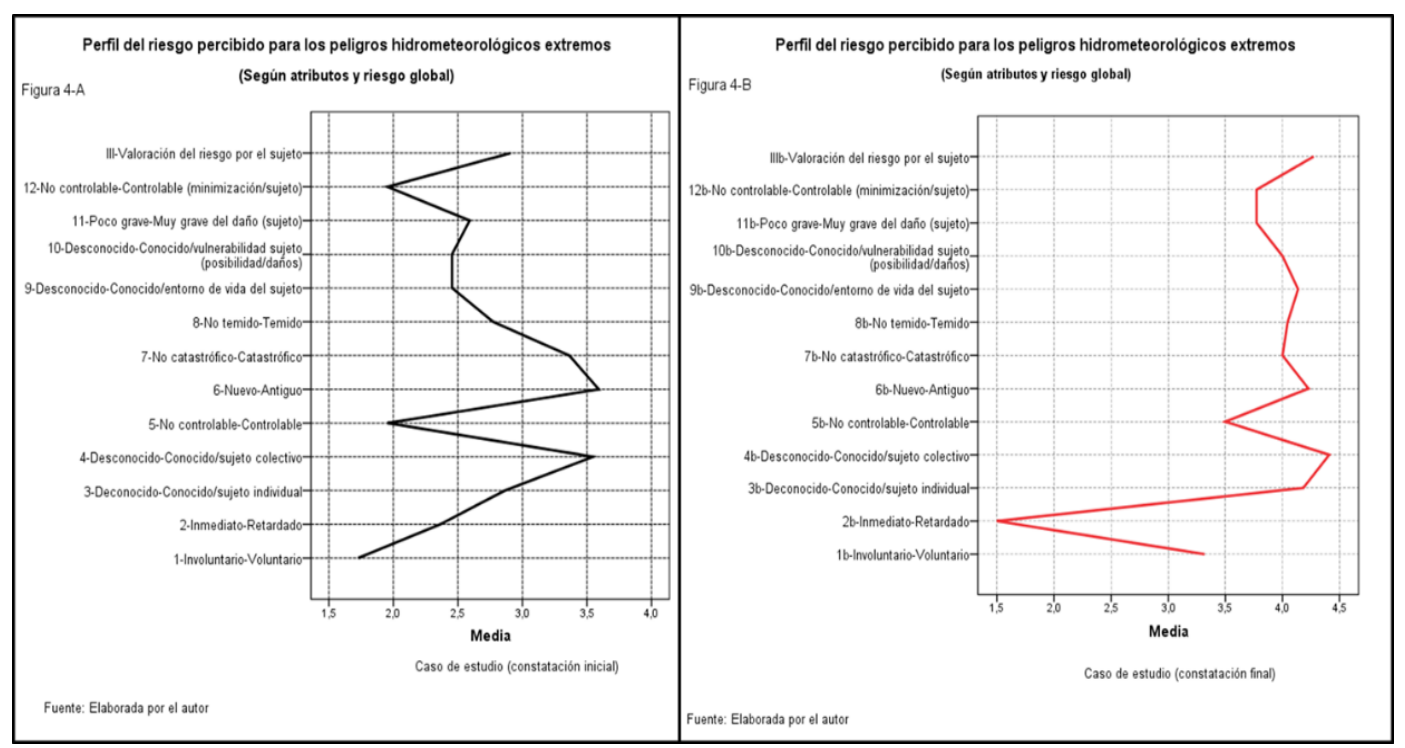

Fuente: Elaboración propia.

Dichos perfiles se corresponden con la Sección /l del instrumento de indagación (encuesta) en la que se:

II-1: Busca conocer la percepción sobre la voluntariedad o involuntariedad en la exposición al peligro por parte del sujeto, en base al reconocimiento de vulnerabilidades individuales y colectivas que repercuten en la gestión de riesgo (involuntariedad-voluntariedad ante el peligro).

II-2: Explora la percepción sobre la demora de las consecuencias, parámetro crítico en el momento de explicar las actitudes y el comportamiento individual y colectivo del sujeto indagado (inmediatez del efecto). 
II-3: Explora el factor conocimiento que tiene el sujeto sobre el peligro (conocimiento del riesgo ante el peligro).

II-4: Explora el factor conocimiento que el sujeto atribuye a los a los responsables de la prevención, en íntima relación con el conocimiento de los responsables, con la confianza en ellos y con la aceptación de las medidas preventivas que se proponen (conocimiento del riesgo por los actores-gestores institucionales o comunitarios).

II-5: Indaga por el grado de control percibido, que permite descartar actitudes fatalistas recurrentes: "pasa cuando pasa y yo no lo puedo evitar»; o por el contrario, sentimientos de excesiva confianza o de invulnerabilidad: "a mí no me sucederá esto porque soy más listo, ya he vivido otros y no es para tanto, tengo mayor experiencia, es más ruido que otra cosa, etc.» (control-personal-sobre el riesgo).

II-6: Explora la percepción del sujeto sobre la novedad o antigüedad del peligro, dado que la familiaridad con el peligro puede generar su no reconocimiento (novedad del riesgo con relación al peligro de estudio).

II-7: Explora el potencial catastrófico que se atribuye al peligro, atributo que mantiene una relación alta y positiva con el riesgo total percibido y su gestión lo más racional y objetivo posible (no catastrófico-catastrófico del peligro).

II-8: Explora la respuesta emocional de temor, la característica más predictiva del peligro y del percibido en general (temido o no temido).

II-9: Explora el grado de conocimiento de la vulnerabilidad del entorno de vida del sujeto (condiciones naturales del lugar: zona baja, paleocauce, cercanía al mar, etc.; acueducto y alcantarillado; estado de la vivienda...), ante el peligro respectivo (conocimiento del entorno y la vulnerabilidad).

II-10: Evalúa la percepción del daño que tiene el sujeto individualmente y que articulada con la pregunta anterior, contribuye a comprender el conocimiento de su vulnerabilidad o susceptibilidad ante el peligro, cuestión central en la gestión del riesgo (posibilidad que experimente el daño).

II-11: Evalúa la percepción de la gravedad de las consecuencias, la que se corresponde con la magnitud de las pérdidas (de vida o materiales), que es una de las variables constitutivas de la definición técnica de riesgo, y se corresponde con la mayor o menor vulnerabilidad del sujeto ante el peligro respectivo (gravedad del daño).

II-12: Trata de explorar tanto la visión que el sujeto tiene de su capacidad para realizar acciones preventivas (reducir la probabilidad de aparición del daño), como de realizar actuaciones para reducir el impacto del daño, lo que abunda en el grado de percepción del peligro por parte del sujeto (autogestión de riesgo del peligro).

La pregunta (sección III) es de tipo general y se dirige a obtener una estimación de la variable magnitud del riesgo percibido. La pregunta incorpora aclaraciones para estandarizar la gravedad de los desenlaces que se deben considerar (pérdidas de salud muy graves) y la latencia (tanto las consecuencias que suponen pérdidas de salud a corto plazo, como a medio o largo plazo). 
La descripción general de los estadígrafos calculados y el perfil para cada atributo de percepción de peligros hidrometeorológicos y del riesgo global -por el sujeto-, para cada momento del curso, revela que tienen valores de grado intermedio los peligros relacionados con el desconocimientoconocimiento, la novedad-antigüedad y la valoración del riesgo. Es importante significar que, según el sujeto, la interacción con el peligro es principalmente «involuntaria» e «incontrolable» para la minimización de los daños a nivel individual y colectivo, lo que denota una actitud de vulnerabilidad cognoscitiva inicial.

Cada atributo fue calculado atendiendo a: sexo, grupo etario (joven-adulto-adulto mayor), nivel de instrucción vencido, situación laboral y tiempo de residencia en el territorio.

Los encuestados reconocen como principal peligro que les afecta al relacionado con las inundaciones por lluvia (63.6\%), contrario a la selección que registra la EPP de 2009-2011, que reconoce al viento; y coincidente con el criterio de los especialistas, como el fenómeno de mayor peligrosidad histórica para la región, cuestión que es percibida por los individuos a través del proceso educativo desarrollado, como parte de la formación de bases cognoscitivas acerca de los peligros hidrometeorológicos en Cuba, en el municipio Mariel de manera particular.

En la constatación realizada al culminar el curso, se percibe mayor nivel de concientización y conocimientos acerca de los peligros hidrometeorológicos extremos, relacionados en primer lugar con el viento, los que afectan al sujeto en mayor o menor medida según las particularidades y contexto relativo; siento las inundaciones por intensas lluvias o las penetraciones del mar, las que ocupan el lugar principal en las percepciones individuales.

De igual forma, se constata empíricamente (constatación inicial) la tendencia generalizada del sujeto al percibir en los grados intermedio, bajo y muy bajo; y por tanto, a la deficiente percepción del riesgo del peligro valorado en los diferentes ámbitos (variables) de análisis, donde se exploran las características siguientes:

- Deficiente reconocimiento de las vulnerabilidades individuales o colectivas que potencian el riesgo respectivo.

- Pobre consideración del potencial destructivo y consecuencias derivadas del fenómeno, con independencia de la relativa confianza en las autoridades e instituciones comunitarias.

- Se registran deficiencias cognoscitivas acerca de los fenómenos hidrometeorológicos, sus efectos y maneras de minimizarlos cuando se producen (no controlable-controlable). En sentido general, existe predisposición acerca del carácter impredecible e inevitable de estos y sus consecuencias, al margen de cualquier control o minimización por parte del sujeto (social).

- En contradicción con lo anterior, el grado de temor es relativamente bajo; quizás condicionado por el contacto histórico con este fenómeno y la relación directa con la alta atención y gestión estatal (institucional, comunitaria, etc.) de protección civil. La población adulta (30-59 años) manifiesta mayor grado de temor inicial cercano al grado intermedio.

- Otro aspecto manifiesto se centra en el grado de familiaridad del individuo con el peligro (II-6; novedad o antigüedad), con la tendencia a la minimización del temor por los mismos, la cual es propiciadora de conductas disímiles ante el fenómeno de estudio.

- Contrario al elemento anterior, es de alto reconocimiento el nivel del daño del fenómeno (II-7; no catastrófico-catastrófico), percepción mayormente diferenciable -en sentido positivo- de la población jubilada y de mayor tiempo de residencia en el territorio. 
- En sentido general, se revela el insuficiente conocimiento de los individuos sobre su entorno de vida cotidiana y sobre las vulnerabilidades sociales y geográficas que le circundan (II-9 y II-10), con excepción de los jubilados y los residentes de 10 años o más en el territorio, precognición dada por experiencia vivida. El resultado inicial no es proporcional con el nivel de instrucción del sujeto, que por demás es alto (Media Superior y Superior), lo que a criterio del autor de la presente investigación, manifiesta insuficiencias educativas en todos los ámbitos (formal, informal, no formal), con independencia de la voluntad política y esfuerzo institucional y de los demás actores-gestores del proyecto social socialista cubano, con más de 56 años de experiencia.

- La mayoría de los encuestados, con independencia de las variables sociodemográficas estudiadas, ve reducida su capacidad para realizar acciones preventivas en aras de reducir la probabilidad de aparición del daño (II-12; autogestión de riesgo del peligro), lo que muestra baja percepción y actitud (social) de enfrentamiento y superación del riesgo como actor individual y colectivo, en el escenario de vida respectivamente.

Los perfiles resultantes de la constatación empírica final muestran resultados superiores al intermedio y alto, y en algunos casos muy alto, para casi todos los atributos de riesgo; en sentido favorable, con relación a la mayor percepción del riesgo por parte del individuo.

Estos resultados reafirman el planteamiento teórico propuesto acerca del impacto de la educación geográfica en la percepción de riesgo por peligros naturales, dado por la contribución del conocimiento de las condiciones geográficas de los espacios de vida cotidiana del sujeto a la formación cultural de la población, en el manejo integrado de sus riesgos previo a la identificación de sus vulnerabilidades.

\section{CONCLUSIONES}

La percepción como acto de naturaleza cognitiva es estudiada desde diversos puntos de vistas, siendo amplia y diversa las investigaciones aplicadas y de autores que se refieren al respecto, con miradas que van desde las perspectivas sicológica y sociológica, aplicada en diferentes ámbitos de la vida social como los intereses de la presente investigación, que se suscribe a la dimensión de la percepción de riesgo, por peligro natural, desde la interacción compleja entre las categorías de espacio natural, espacio económico y de hábitat, espacio social y espacio cultural, de los sujetos sociales (individual y colectivo), en torno a los peligros naturales contextualmente.

La incorporación de la perspectiva geográfica en el análisis del riesgo por peligros hidrometeorológicos extremos -como proceso construido social y culturalmente- contribuye a poner de manifiesto las profundas interconexiones entre el entorno socioeconómico, político, ambiental y cultural, generado en una región o comunidad, así como a distinguir los procesos y los niveles de vulnerabilidad geográfica del entorno de vida social que lo condicionan, lo cual contribuye a minimizar la visión positivista fragmentada del mundo.

El conocimiento geográfico como saber cultural, en armonía con la identidad local como patrimonio personal, contribuye a desarrollar la subjetividad y la percepción como base de la actitud ciudadana de responsabilidad social, en el fomento de una cultura de prevención y adaptación al cambio climático y del desarrollo local ambientalmente sostenible. 


\section{REFERENCIAS BIBLIOGRÁFICAS}

Agencia de Medio Ambiente. (2013). Base de Datos Nacional sobre percepción de riesgo por peligros hidrometeorológicos 2009-2011. [Grupo Nacional de Evaluación de Riesgos- Agencia de Medio Ambiente]

Almaguer, C. (2008). El riesgo de desastres: una reflexión filosófica [Tesis doctoral, Universidad de La Habana]. https://cutt.ly/NuQfJrc

Bayón, P. (2014). Cultura ambiental: educación y ética para su formación. En: Cooperativismo, producción y reproducción de la vida y cultura ambiental. Editorial Camino

Castañeda, G. (2014, del 23 al 24 de marzo). Percepción del riesgo a huracanes en cinco comunidades costeras del estado de Colima [ponencia]. 4to Congreso Nacional de ciencias Sociales, Chiapas, México.

Conesa, C., y Calvo, F. (2003). Los procesos de riesgo con origen natural: una constante en la relación entre hombre y medio. Revista Internacional de Ciencias Sociales, (23), 5-15. https://revistas.um.es/areas/article/view/117851

Espluga, J. (2002, 27 de junio). Las dimensiones psicológicas, sociales y culturales del riesgo y su relación con la prevención de riesgos laborales. Jornada Técnica: La nueva cultura de la prevención: De la culpabilidad al compromiso. Barcelona, España.

Fischoff, E., Slovic, P., \& Lichtenstein, M. (1979). Weighing the Risks: Risks: Benefits which Risks are Acceptable? Environment: Science and Policy for Sustainable Development, 21(4), 17-38, https://doi.org/10.1080/00139157.1979.9929722

Lobato, R. (1995). Espaço: um conceito-chave da Geografia. No IE Castro, PC Costa y R Lobato (Eds.), Geografia: conceitos e temas (2 ${ }^{\text {da }}$ adicione, pp. 15-48). Bertrand Brasil.

Mateo, J. (2001). Geografía de los paisajes. Primera parte. Paisajes naturales. Editorial Félix Varela.

Puy, A. (1994). Percepción social del riesgo. Dimensiones de predicción y evaluación [Tesis doctoral, Universidad Complutense]. https://eprints.ucm.es/3002/1/T18978.pdf

Puy, A., y Aragonés, J. (1997). Percepción social de los riesgos y gestión de las emergencias ambientales. Desastres y Sociedad, (8), 39-58. https://url2.cl/uXB4L

Slovic, P., \& Weber, E. (2002, April 12-13). Perception of Risk Posed by Extreme Events. In Risk Management strategies in an Uncertain World Palisades, New York, United States of America. https://url2.cl/uXB4L

Unión Geográfica Internacional/Comisión de Educación Geográfica. (1992). Declaración Internacional sobre Educación Geográfica. https://url2.cl/rZYMW 